6 months. Agalsidase alfa was generally well tolerated and had a safety profile in Fabry disease patients with ESRD similar to that observed in patients without ESRD. Infusionrelated reactions (e.g. chills, fever, headache, flushing) occurred in $38.1 \%$ of patients, but did not cause any premature discontinuations of treatment. Neither of two deaths (due to pneumonia plus arrhythmia, and lung malignancy) was attributed to enzyme replacement therapy. Pharmacokinetic studies revealed the typical biphasic serum elimination profile. Mean serum clearance profiles and pharmacokinetic parameters were similar in transplant and dialysis patients with Fabry disease and ESRD, and were also similar to those of a group of 18 non-ESRD patients with the condition.

Original article Pastores GM et al. (2007) Safety and pharmacokinetics of agalsidase alfa in patients with Fabry disease and end-stage renal disease. Nephrol Dial Transplant 22: 1920-1925

\section{Machine-generated bicarbonate dialysate customized for patients with acute renal failure}

Over a decade ago, a group from the Section of Dialysis and Extracorporeal Therapy at The Cleveland Clinic, $\mathrm{OH}$, developed a bicarbonate-based dialysate for use during continuous renal replacement. An adapted intermittent hemodialysis machine measures and mixes acid-bicarbonate and ultra-pure water into dialysate in desired quantities. Bicarbonate-based dialysate can more efficiently correct acid-base disturbances in patients with acute renal failure (ARF) and does not induce some of the adverse effects associated with lactate-based fluids.

Customized bicarbonate-based dialysate is now the primary source for patients with ARF treated in the clinic's intensive care units. Teo et al. have analyzed data from the CCFARF Support Registry and have identified 405 patients initially supported with continuous bicarbonate hemodialysis during the period 1994-2004-an average of 1,292 days of hemodialysis per year.

At $60.5 \%$, intensive care unit mortality was similar to that observed in other series. Blood urea nitrogen, creatinine, serum electrolytes, glucose and bicarbonate levels were all well controlled throughout therapy. Dialysate yielded no positive cultures and did not contain raised levels of endotoxins. The cost of dialysate production decreased during the course of the study, from US\$0.91// in 1995 to $\$ 0.67 /$ in 2005 ; customized pharmacy preparations would cost approximately $\$ 14.00 /$. All patients were supported with variablecomposition dialysate without experiencing any adverse effects. The authors deduce that their machine-generated dialysate, which can be customized and produced in large volumes, is safe, effective and economic for continuous hemodialysis in patients with ARF.

Original article Teo BW et al. (2007) Machine-generated bicarbonate dialysate for continuous therapy: a prospective, observational cohort study. Nephrol Dial Transplant 22: 2304-2315

\section{Intraperitoneal electrolytes in peritoneal dialysis: an alternative to oral supplements?}

Hypokalemia and hypomagnesemia are occasional complications of peritoneal dialysis. Oral supplementation of potassium and magnesium might not be effective as a result of patient noncompliance and chronic gastrointestinal losses. In a recent paper, Amirmokri and colleagues have reported the use of oral and intraperitoneal administration of these key ions in 27 stable patients on continuous cyclic peritoneal dialysis for whom 1 year of laboratory data were available.

Among the study population, 15 patients were receiving oral potassium supplementation, and 10 were receiving oral magnesium. Three patients had persistent hypokalemia and hypomagnesemia despite oral supplementation. In total, five patients received one intraperitoneal dose of potassium and/or magnesium $(4 \mathrm{~g}$ $\mathrm{MgSO}_{4}$ and/or $60-80 \mathrm{mmol} \mathrm{KCl} \mathrm{per} 2 \mathrm{l}, 2.5 \%$ peritoneal dialysis bag; used as last dwell). Oral supplementation was discontinued in these patients.

Serum potassium and magnesium concentrations normalized in all five patients within 6-8h of intraperitoneal administration (potassium: mean serum level $3.2 \mathrm{mmol} / \mathrm{l}$ at baseline, $4.1 \mathrm{mmol} / \mathrm{l}$ at $6-8 \mathrm{~h}$; magnesium: mean serum level $0.62 \mathrm{mmol} / /[1.5 \mathrm{mg} / \mathrm{dl}]$ at baseline, $1.07 \mathrm{mmol} / \mathrm{l}[2.6 \mathrm{mg} / \mathrm{dl}]$ at $6-8 \mathrm{~h}$ ), with the effects lasting for up to $20-24 \mathrm{~h}$. Treatments were well 